\title{
Morphometrical Analysis on Topographical Difference in Size Distribution, Number and Volume of Islets in the Human Pancreas
}

\author{
Ken Saito, Noriyuki Imama and Tohru Takahashi \\ First Department of Pathology, Tohoku University School of \\ Medicine, Sendai 980
}

\begin{abstract}
Samto, K., Iwama, N. and Takahashu, T. Morphometrical Analysis on Topographical Difference in Size Distribution, Number and Volume of Islets in the Human Pancreas. Tohoku J. exp. Med., 1978, 124 (2), 177-186 - Seven pancreases from nondiabetic autopsy cases were submitted to morphometrical analysis of the islets of Langerhans as a preliminary investigation of the diabetic pancreas. The pancreatic islets were approximately simulated by spheres of different sizes randomly dispersed in the three-dimensional space; thereon the parameters of Weibull function assumed for the distribution of islet radii were estimated stereologically from chord length measurements on histologic sections taken from different portions of the pancreas. By means of the estimated parameters topographical comparison of the number, volume, mean radius and size distribution of islets in a unit volume was performed. The islet distribution appeared to change the pattern gradually according to the position from the pancreatic head to the tail: the head was characterized by abundance of small islets that corresponded to a slightly higher islet population thereat, whereas the tail by moderate increase of larger islets that caused a steep increase of islet volume here. To estimate parameters for the whole pancreas a histologic section from a range between the midposition of the pancreas and the one-fourth position from the splenic end was regarded to be a fairly good representative. - pancreatic islets; spherical bodies; size distribution function; parameters; topographical comparison
\end{abstract}

The pancreatic islets are regarded as a group of spherical or ellipsoidal bodies of different sizes randomly dispersed in the three-dimensional space. In quantitation of the islets in the pancreas, as has been stressed by some authors (Lazarus and Volk 1962; Warren et al. 1966), it is impossible to make an adequate estimation immediately from observations of distribution of islet sections on a histologic slide.

Using a large number of alternate serial sections from a monkey pancreas, Thompson (1932) and Thompson and Hussey (1932) made an attempt at analyzing the density and frequency distribution of the volume of the three-dimensional structures for which they did not consider any appropriate geometrical model. For assessment of the population density they measured the diameter of randomly

Received for publication, July 7, 1977.

This study was supported in part by a grant-in-aid (087012) from the Ministry of Education, Science and Culture, Japan. 
selected islets in the rectangular direction to the pancreatic sections, and for the islet volume determination integrated the areas of all islet sections belonging to the same islet by means of planimetry. The method was applicable only to a limited number of cases, because it took much time.

Wicksell $(1925,1926)$, a pioneer of the corpuscle problem, derived a mathematical relation correlating the distribution of sphere diameters to that of circle diameters on a test plane to examine the distribution of Malpighian follicles in the spleen; further he extended the correlation to the case of ellipsoids. A few decades later Hellman $(1959 \mathrm{a}, \mathrm{b})$ applied the equation system derived by Wicksell to the human pancreatic islets, and showed extremely skew curves of the islet distribution with the highest incidence of the smallest diameters in all cases examined. Regards to their skewness, however, the size distribution curves calculated were not brought to precise comparison between individual cases with appropriate parameters.

Suwa et al. (1964) first attempted in their analysis of cirrhotic nodules of the liver to estimate the parameters of a theoretical function assumed for the sphere distribution from measurements of the length of chords delivered by interception of a test line with the sphere group. In a recent report of Suwa et al. (1976), various methods for estimation of the sphere distribution were discussed; and the parameter estimation with the chord length was shown to be preferable to that with the circle diameter at least for the pancreatic islets. It is because the error of the estimates caused by omission of small and indiscernible islet sections was minimized in the former method.

Since Opie (1900) it has been reported frequently that there would be more numerous islets in the tail than in the head and body of the pancreas. However, the conclusion does not seem to be substantiated by a reliable quantitative method. In the present report the topographical difference in the islet distribution is examined with some pertinent parameters. This is a preliminary investigation for morphometrical analysis on the pancreatic islets in the human diabetics.

\section{Materials and Methods}

The material consists of the pancreases from 7 autopsy cases of nondiabetic adults. They all showed normal blood sugar level and negative urinary sugar (Table 1). After 10\% formalin fixation the organs were removed of peripancreatic adipose tissue and larger blood vessels and their volumes were measured by means of water replacement; then the whole pancreas was cut into $3 \mathrm{~mm}$ thick sagittal slices which were numbered with the increasing numerals from the slice adjacent to the papilla of Vater to that of the splenic end. The position $\mathrm{x}$ of the slice under examination was indicated with the decimal subscript equal to its own numeral divided by the total number of slices (Fig. 1).

A $3 \mu \mathrm{m}$ thick paraffin section was obtained from each of the slices to examine and stained with the modified Masson's trichrome method substituting Biebrich scarlet for acid fuchsin.

Fig. 2 illustrates the geometrical relation of chord length to sphere radii at the interception of a random test line. With a horizontal scale of the eye-piece as the test line (see Fig. 3) the chord length was successively measured by sliding the histologic section transversely under 200-fold magnification. Including interlobular connective tissue, blood vessles and larger pancreatic ducts the whole pancreatic section was scanned with parallel 
Table 1. Cases examined

\begin{tabular}{|c|c|c|c|c|c|}
\hline $\begin{array}{l}\text { Case } \\
\text { No. }\end{array}$ & $\begin{array}{l}\text { Age and } \\
\text { sex }\end{array}$ & $\begin{array}{c}\text { Pancreatic } \\
\text { volume }\left(\mathrm{cm}^{3}\right)\end{array}$ & $\begin{array}{l}\text { Number of } \\
\text { slices }\end{array}$ & $\begin{array}{l}\text { Postmortem } \\
\text { time (hr:min) }\end{array}$ & Pathological diagnosis \\
\hline 1 & 21 우 & 60.5 & 34 & $1: 20$ & Myeloproliferative disorder \\
\hline 2 & $23+$ & 58.5 & 40 & $2: 15$ & Posthemorrhagic collapse \\
\hline 3 & 49 今 & 52.3 & 54 & $2: 10$ & Bronchial carcinoma \\
\hline 4 & 54우 & 40.1 & 38 & $1: 30$ & $\begin{array}{l}\text { Mammary carcinoma, radiation } \\
\text { pneumonitis }\end{array}$ \\
\hline 5 & 64 今 & 64.9 & 40 & $1: 12$ & $\begin{array}{l}\text { Liver cirrhosis, rupture of esophageal } \\
\text { varices }\end{array}$ \\
\hline 6 & 66 今 & 72.4 & 44 & $1: 30$ & $\begin{array}{l}\text { Pulmonary carcinoma, organized } \\
\text { pneumonia }\end{array}$ \\
\hline 7 & 73 今 & 60.4 & 40 & $2: 45$ & $\begin{array}{l}\text { Pulmonary carcinoma, organized } \\
\text { pneumonia }\end{array}$ \\
\hline
\end{tabular}

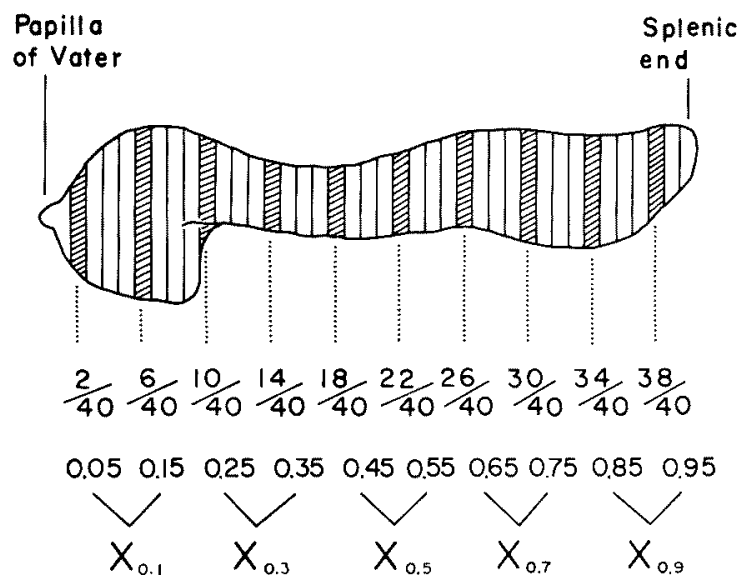

Fig. 1. Case $2(23 \delta)$. The whole pancreas was sectioned to 40 sagittal slices which were numbered with increasing numerals from the duodenal tip to the splenic end. The positions x's of 10 slices under examination, hatched in the illustration, were indicated with decimal subseripts dividing their original numerals by the total slice number. For further detail see text.

test lines at an interval of approximately $0.3 \mathrm{~mm}$, which was not smaller than the diameter of the largest iselt section. To obtain a sufficiently large number of chords two histologic sections from two adjacent slices, as illustrated in Fig. 1, were used for measurement at each position designated. For the estimation of a quantity in regard to the whole pancreas, the measurements on all examined sections were pooled and weighted by the dimension of the area of individual pancreatic sections, which was planimetrically determined on their projection images.

In the treatment hereafter possible errors due to tissue contraction through the process of paraffin embedding were disregarded.

\section{Mathematical Treatment}

As derived in the report of Suwa et al. (1976), the general relation to correlate the distribution $N(r)$ of sphere radius $r$ with the distribution $F(\lambda)$ of chord length $\lambda$ is expressed by 


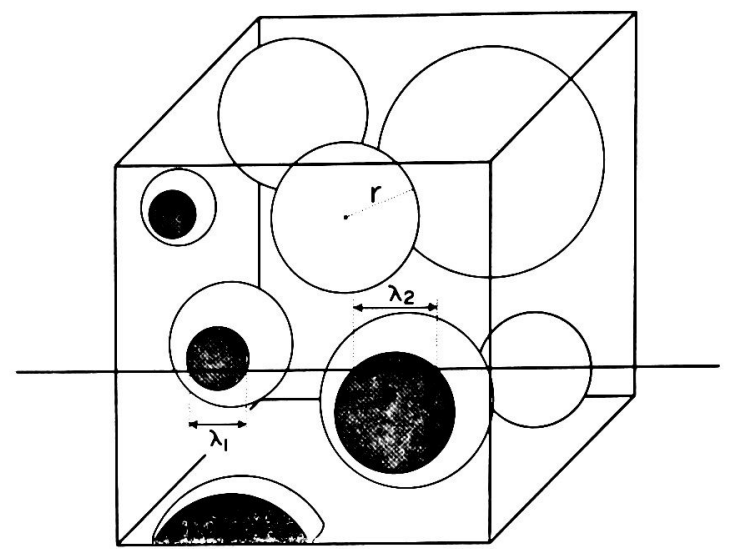

Fig. 2. The pancreatic islets were approximately simulated by a geometrical model of spheres of different radii in the three-dimensional space. Suppose a sufficiently long straight line randomly penetrating the space, and a number of chords of length $\lambda$ are delivered by interception of the line with the sphere group. The relation can be practically reproduced by interception of a randomly drawn test line with circular sections (shaded areas) on a random histologic section.

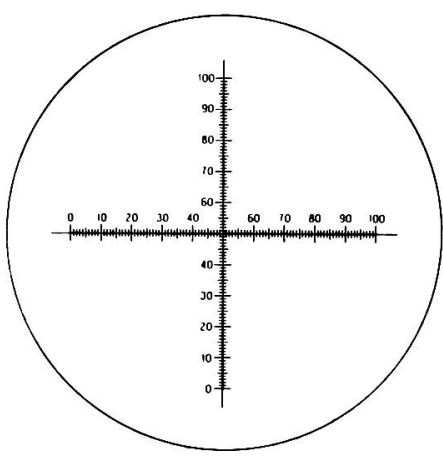

(a)

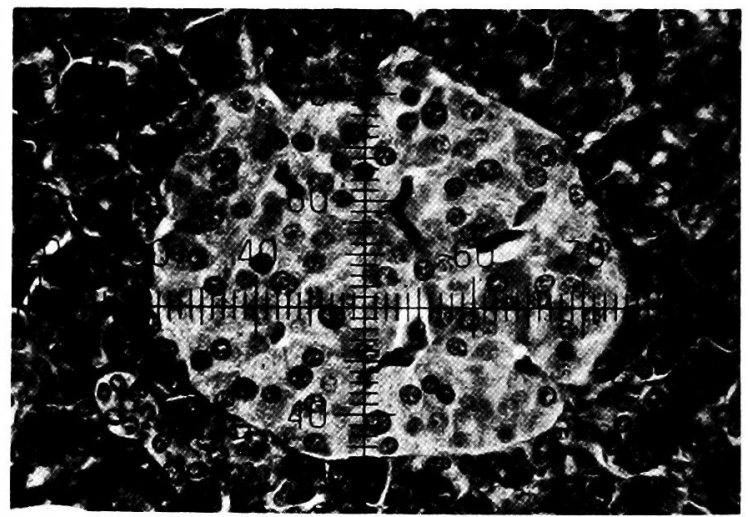

(b)

Fig. 3 (a) Eye-piece with two crossed scales. (b) With the horizontal scale as a test line the chord length is successively measured by sliding a histologic section transversely under 200-fold magnification. The vertical scale is utilized to determine the interval of test lines. Modified Masson's trichrome stain.

$$
F(\lambda)=\frac{\pi}{2} \lambda \int_{\lambda / 2}^{\infty} N(r) \mathrm{d} r .
$$

Accordingly, the following integration $I_{n}(\lambda)$ is considered,

$$
\begin{aligned}
I_{n}(\lambda) & =\int_{0}^{\infty} \lambda^{n} F(\lambda) \mathrm{d} \lambda=\frac{\pi}{2} \int_{0}^{\infty} \int_{\lambda / 2}^{\infty} \lambda^{n+1} N(r) \mathrm{d} r \mathrm{~d} \lambda \\
& =\frac{2^{n+1} \pi}{n+2} \int_{0}^{\infty} r^{n+2} N(r) \mathrm{d} r
\end{aligned}
$$

where $n$ is 0 or a positive integer; and the next equations are immediately obtained by definition: 


$$
I_{0}(\lambda)=N_{\lambda_{0}}, I_{1}(\lambda) / I_{0}(\lambda)=\bar{\lambda}, I_{2}(\lambda) / I_{0}(\lambda)=\left(\overline{\lambda^{2}}\right) .
$$

In the above expressions $N_{\lambda \theta}, \bar{\lambda}$ and $\left(\overline{\lambda^{2}}\right)$ are the number, the arithmetic mean and the second moment of chord length per test line of unit length, respectively. In the present study the following three theoretical functions were assumed for sphere radius distribution:

Weibull distribution

$$
N(r)=N_{v o}\left(m / r_{0}\right)\left(r / r_{0}\right)^{m-1} \exp \left[-\left(r / r_{0}\right)^{m}\right]
$$

Gamma distribution

$$
N(r)=N_{v o}\left(1 / r_{0} \Gamma(m)\right)\left(r / r_{0}\right)^{m-1} \exp \left(-r / r_{0}\right),
$$

Logarithmic normal distribution

$$
N(r)=N_{v o}(1 / \sqrt{2 \pi} m r) \exp \left[-\left(\log r-\log r_{0}\right)^{2} / 2 m^{2}\right],
$$

where $N_{v o}, m$ and $r_{0}$ denote the number of spheres in a unit volume, the parameter for the geometrical pattern of distribution and the scalar parameter, respectively. From measured $N_{\lambda_{0}}, \bar{\lambda}$ and $\left(\overline{\lambda^{2}}\right)$ all the above parameters were determined by means of (3). Then, the expected value of the fourth moment of chord length or $E\left(\overline{\lambda^{4}}\right)$ was calculated with the estimated parameters using (2). Its ratio to observed $\left(\overline{\lambda^{4}}\right)$ reveals the grade of adaptation of an assumed theoretical distribution.

The volume ratio $V_{0}$ of the pancreatic islets is given by the sum $\sum \lambda$ of length of chords per test line of unit length.

For an example, measurements on histologic sections from slices 46 and 52 of case 3 are shown:

$$
\begin{aligned}
& L \text { (entire length of test line })=867.915 \mathrm{~mm} \\
& N_{\lambda} \text { (total number of chords) }=417 \\
& N_{\lambda_{0}}=0.48046 / \mathrm{mm} \\
& \bar{\lambda}=0.07862 \mathrm{~mm} \\
& \left(\overline{\lambda^{2}}\right)=0.008681 \mathrm{~mm}^{2} \\
& \left(\overline{\lambda^{4}}\right)=0.00020311 \mathrm{~mm}^{4}
\end{aligned}
$$

Estimated parameters for the three theoretical distribution functions together with $\bar{r}$ and $\mathrm{E}\left(\overline{\lambda^{4}}\right) /\left(\overline{\left.\lambda^{4}\right)}\right.$ are listed in the following table. $N(r)$ 's calculated are conspicuously different in the region of small $r$.

Weibull distribution

$$
\begin{aligned}
& m=1.2469 \\
& r_{0}=0.0282 \mathrm{~mm} \\
& \bar{r}=0.0263 \mathrm{~mm} \\
& N_{v \sigma}=134.30 / \mathrm{mm}^{3} \\
& \mathrm{E}\left(\overline{\lambda^{4}}\right) /\left(\overline{\lambda^{4}}\right)=1.009
\end{aligned}
$$

$$
\begin{aligned}
& \text { Logarithmic normal } \\
& \text { distribution } \\
& m=0.4713 \\
& r_{0}=0.0338 \mathrm{~mm} \\
& \bar{r}=0.0378 \mathrm{~mm} \\
& N_{y 0}=85.66 / \mathrm{mm}^{3} \\
& \mathbf{E}\left(\overline{\lambda^{4}}\right) /\left(\overline{\lambda^{4}}\right)=1.203
\end{aligned}
$$

For assessment of the adaptability of three assumed distributions, $F(\lambda)$ 's were calculated by (1) with the estimated parameters, and compared with measured distribution of $\lambda$. Weibull and gamma distributions fitted fairly well to observed distribution in comparison with logarithmic normal distribution, particularly in the region of smaller $\lambda$. The impression was justified by the ratio $\mathbf{E}\left(\overline{\lambda^{4}}\right) /\left(\overline{\lambda^{4}}\right)$ that was approximately 1 in the former two distributions. With the pancreatic islets the advantage of the two distributions was generally noticed in all the measurements. Gamma function was, however, inapplicable to two cases of extremely skew chord length distributions.

After this preliminary examination Weibull distribution was adopted for the estimation of the islet distribution on account of its good adaptability. 


\section{Results}

The number, volume and mean radius of islets

The number $N_{v o}$ of islets in a unit volume of the pancreas was somewhat larger at the position $x_{0.1}$ than the numbers at the other positions (Fig. 4, Table 2), and a statistically significant difference was found between $N_{v o}\left(\mathrm{x}_{0.1}\right)$ and $N_{v o}\left(\mathrm{x}_{0.7}\right)$.

TABLE 2. Arithmetic moans of the estimated parameters

\begin{tabular}{llllll}
\hline \multicolumn{1}{c}{$\begin{array}{c}\text { Means of } \\
\text { parameters }\end{array}$} & \multicolumn{1}{c}{$\mathrm{x}_{0.1}$ (Head) } & \multicolumn{1}{c}{$\mathrm{x}_{0.5}$} & \multicolumn{1}{c}{$\mathrm{x}_{\mathbf{0 . 7}}$} & \multicolumn{1}{c}{$\mathrm{x}_{0.9}$ (Tail) } & \multicolumn{1}{c}{ Pooled } \\
\hline $\bar{V}_{o} \mathrm{~mm}^{3} / \mathrm{mm}^{3}$ & $0.0139 \pm 0.0036$ & $0.0146 \pm 0.0047$ & $0.0174 \pm 0.0061$ & $0.0228 \pm 0.0088$ & $0.0163 \pm 0.0048$ \\
$\bar{N}_{v o} / \mathrm{mm}^{3}$ & $209.31 \pm 162.49$ & $138.41 \pm 80.44$ & $132.37 \pm 82.43$ & $135.32 \pm 59.80$ & $159.26 \pm 97.76$ \\
$\hat{r} \mathrm{~mm}$ & $0.0133 \pm 0.0036$ & $0.0160 \pm 0.0042$ & $0.0182 \pm 0.0036$ & $0.0206 \pm 0.0057$ & $0.0157 \pm 0.0035$ \\
$\bar{m}$ & $0.8658 \pm 0.0988$ & $0.9244 \pm 0.1286$ & $1.0212 \pm 0.1408$ & $1.0805 \pm 0.1454$ & $0.9266 \pm 0.0989$ \\
$\bar{r}_{0} \mathrm{~mm}$ & $0.0124 \pm 0.0041$ & $0.0154 \pm 0.0051$ & $0.0184 \pm 0.0045$ & $0.0212 \pm 0.0067$ & $0.0152 \pm 0.0041$ \\
\hline
\end{tabular}

Statistical significance ( $t$-distribution, $a<0.05$ )

$$
\begin{aligned}
& \bar{V}_{o}: \quad \mathrm{x}_{0.1}, \mathrm{x}_{0.5}<\text { Pooled, } \mathrm{x}_{0.7}<\mathrm{x}_{0.9} \quad \bar{m}: \mathrm{x}_{0.1}, \mathrm{x}_{0.5}, \text { Pooled }<\mathrm{x}_{0.7}, \mathrm{x}_{0.9}\left(\mathrm{x}_{0.1}<\text { Pooled }\right) \\
& \bar{N}_{v_{0}}: \quad \mathrm{x}_{0.1}>\mathrm{x}_{0.7} \\
& \left.\dot{r}: \mathrm{x}_{0.1}<\text { Pooled, } \mathrm{x}_{0.5}, \mathrm{x}_{\mathbf{0 . 7}}, \mathrm{x}_{0.9} \quad \mathrm{x}_{0.5}<\mathrm{x}_{0.9}\right) \\
& \text { (Pooled }<\mathrm{x}_{0.7} ; \mathrm{x}_{0.5}<\mathrm{x}_{0.9} \text { ) }
\end{aligned}
$$

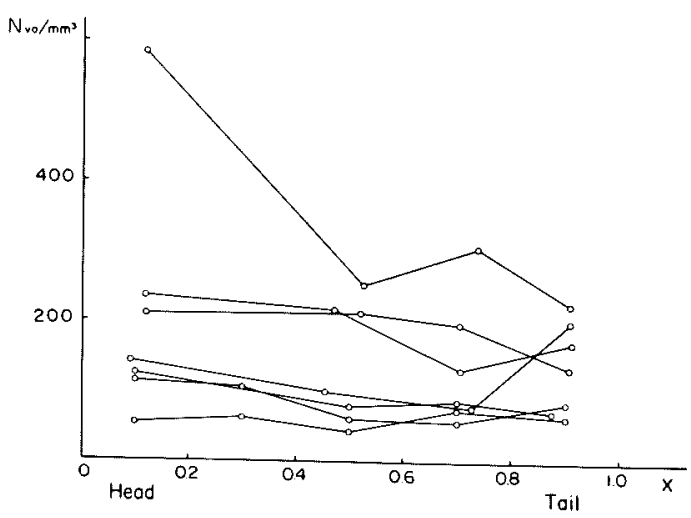

Fig. 4. The islet number $N_{v o}$ per $\mathrm{mm}^{3}$ pancreatic tissue. Note a remarkable variation of $N_{v o}$ at each position of individual pancreases. See also Table 2.

The islet volume $V_{o}$ per unit volume of the pancreas was found to increase gradually from $x_{0.1}$ to $x_{0.7}$, and quite steeply at $x_{0.9}$ (Fig. 5, Table 2). The arithmetic mean $\bar{V}_{o}$ (pooled) of $V_{o}$ (pooled) calculated with regard to the whole pancreas showed an intermediate value between $\bar{V}_{o}\left(\mathrm{x}_{\mathbf{0 . 5}}\right)$ and $\bar{V}_{o}\left(\mathrm{x}_{0.7}\right)$.

The mean radius $\bar{r}$ of islets also showed a steady rise from $x_{0.1}$ to $x_{0.9}$ approximately parallel to the increase of islet volume (Fig. 6, Table 2). The arithmetic mean $\hat{r}$ (pooled) of mean radius $\bar{r}$ (pooled) in the seven pancreases was almost equal to $\hat{r}\left(\mathbf{x}_{\mathbf{0 . 5}}\right)$. 


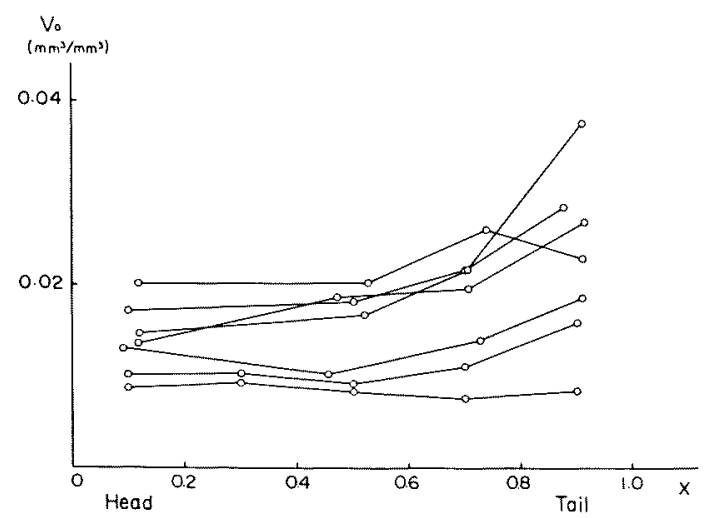

Fig. 5. The islet volume $V_{o}$ per $\mathrm{mm}^{3}$ pancreatic tissue. A steep increase of $V_{0}$ at the position $x_{0.9}$ is noted. See also Table 2.

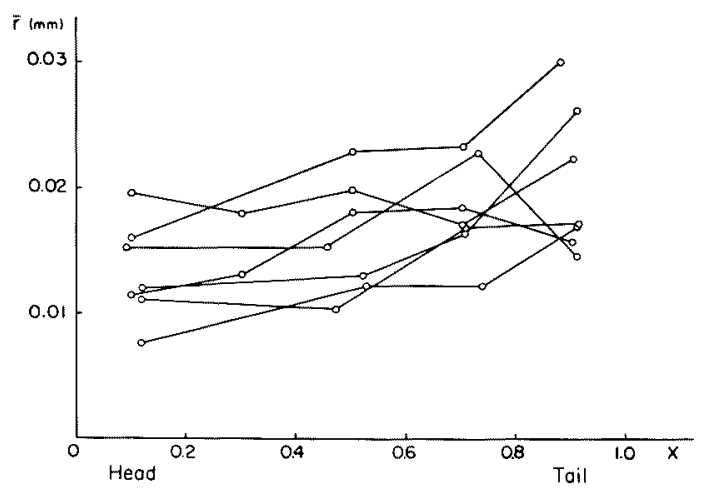

Fig. 6. The mean radius $\bar{r}$ of islets. Note a gradual increase of $\bar{r}$ from $\mathrm{x}_{0.1}$ to $\mathrm{x}_{0.9}$, approximately parallel to the increase of $V_{o}$ in Fig. 5.

Geometrical pattern of the distribution of islet radii

Geometrical pattern of the islet distribution is primarily determined by parameter $m$. Its value rose gradually from $x_{0.1}$ to $x_{0.9}$ (Table 2 ). The arithmetic mean $\bar{m}$ (pooled) for the whole pancreas showed a value slightly larger than $\bar{m}\left(\mathrm{x}_{\mathbf{0 . 5}}\right)$.

On the other hand, a definite positive correlation was noticed between the geometrical parameter $m$ and the scalar parameter $r_{0}$. The regression equation was given by

$$
m=26.2498 r_{0}+0.5287
$$

(correlation coefficient: 0.9551 )

From $\bar{m}$ and $\bar{N}_{y o}$ of Table 2 by using (7), $N(r)$ 's were calculated for the different positions.

All the four curves show a conspicuously skew pattern (Fig. 7). At the position $\mathrm{x}_{0.1}$ extremely high incidence of $r$ smaller than $0.02 \mathrm{~mm}$ is noted, while at $\mathrm{x}_{\mathbf{0 . 9}}$ incidence of larger $r$ is moderately increased, which would contribute much to 


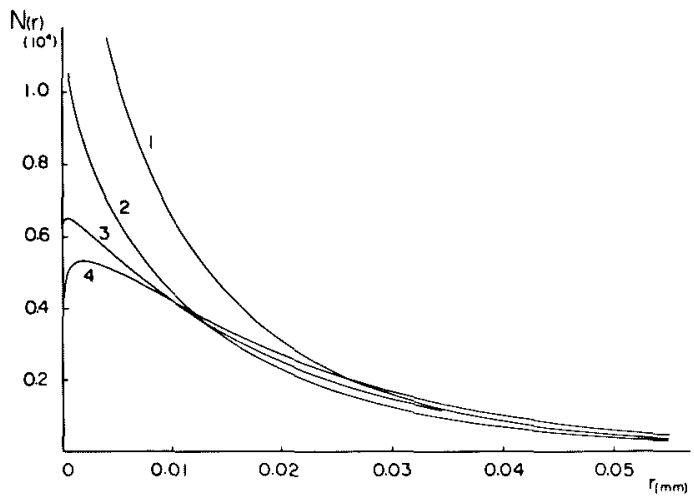

Fig. 7. The distributions of islet radii at different positions of the pancreas calculated with the arithmetic means of parameters listed in Table 2.1 At the position $\mathbf{x}_{0.1}$ (the head). 2 At $x_{0.5}$. 3 At $x_{0.7} .4$ At $x_{0.9}$ (the tail). All the four curves show remarkable skewness. Note extreme abundance of small islets at $x_{0.1}$, in contrast suppressed decline of incidence of larger islets at $\mathbf{x}_{0.9}$.

TABLE 3. Total volume, number and mean radius of islets in the pancreas

\begin{tabular}{cccc} 
Case No. & $\begin{array}{c}\text { Islet } \\
\text { volume }\left(\mathrm{cm}^{3}\right)\end{array}$ & $\begin{array}{c}\text { Islet } \\
\text { number }\left(\times 10^{6}\right)\end{array}$ & $\begin{array}{c}\text { Mean } \\
\text { radius }(\mathbf{m m})\end{array}$ \\
\hline 1 & 1.095 & 12.608 & 0.0121 \\
2 & 0.638 & 3.630 & 0.0185 \\
3 & 1.077 & 9.104 & 0.0165 \\
4 & 0.878 & 14.796 & 0.0105 \\
5 & 1.311 & 6.010 & 0.0215 \\
6 & 0.977 & 8.689 & 0.0168 \\
7 & 0.544 & 5.356 & 0.0143 \\
\hline
\end{tabular}

the steep increase of $V_{o}$ at this position, because the islet volume increases in proportion to the third power of its radius.

The total number and volume of islets

In the seven nondiabetic pancreases the total number of islets varied within the range of $3.630 \times 10^{6}$ to $14.796 \times 10^{6}$; the value of the total islet volume also showed a remarkable variation between $0.544 \mathrm{~cm}^{3}$ to $1.311 \mathrm{~cm}^{3}$ (Table 3). No definite correlation was found between the two quantities.

\section{Discussions}

Islet radius distribution curves estimated in the present study all showed an extremely skew pattern with high incidence of radii smaller than $0.02 \mathrm{~mm}$ (Fig. 7). Hellman (1959a) calculated relative frequency distributions of islets of different size classes using the equation system derived by Wicksell $(1925,1926)$ and also obtained asymmetrical curves. In his study, however, the number of islets of radii smaller than $0.023 \mathrm{~mm}$ was not taken into consideration, and all 


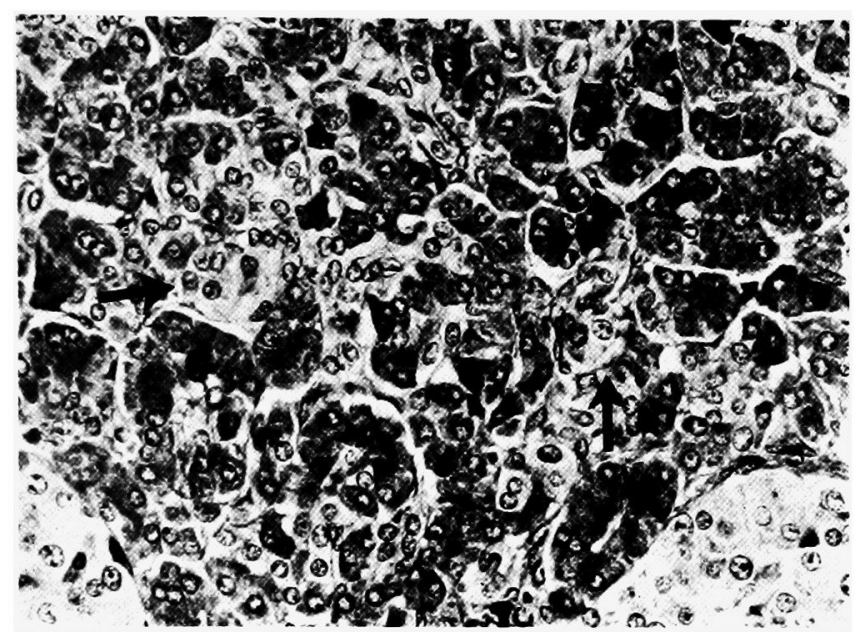

Fig. 8. Two insular buds (arrows) in connection with intercalated ductules are demonstrated under 200-fold magnification. Modified Masson's trichrome stain.

islet sections with diameters smaller than $0.042 \mathrm{~mm}$ (10 mm at 240 -fold magnification) were excluded from measurement. In fact, however, an unexpectedly large number of small islet sections are observed on precise inspection over a pancreatic section; further, as described by Neubert (1927), insular buds in connection with pancreatic ductules composed of a single cell or a few with the specific granules are often recognized (Fig. 8). These were all included in the present measurements.

The islet number per $\mathrm{mm}^{3}$ pancreatic volume varied with a wide range of 43.26 to 583.42 (Fig. 4). The value is roughly several times larger than the range of 10.6 to 43.8 reported by Hellman (1959a). The discrepancy would be principally due to the above-mentioned exclusion of small islets which constitute the majority of islet population in the latter report. On the other hand, the islet number in this study might be overestimated to some extent. As noticed in Fig. 7, $N(r)$ was estimated at a very high level even in the region of $r$ near 0 . It is clear that islets smaller than a single insular cell can not be present. However, no correction was made in the present study. For this problem it would be necessary to define geometrical shape and size of an insular cell. Maclean and Ogilvie (1955) proposed the islet number with a range of 6.4 to 23.9 per $\mathrm{mg}$ pancreatic weight by assuming a group of spheres of a uniform (medium-sized) radius for the islets. As already mentioned, such an assumption does not seem to be adequate in the case of pancreatic islets.

The islet volume per $\mathrm{mm}^{3}$ pancreatic tissue ranged from 0.0077 to $0.0378 \mathrm{~mm}^{3}$ and showed a progressive increase from the duodenal to splenic end (Fig. 5; Table 2). A similar topographical trend was reported by Hellman (1959a) with 5 nondiabetic human pancreases and by Acosta et al. (1969) with 11 normal pancreases of adult mongrel dogs.

Topographical differences in the estimated parameters reflect the changes in the pattern of islet distribution from the duodenal portion $\mathrm{x}_{\mathbf{0 . 1}}$ to the splenic end 
$\mathrm{x}_{0.9}$ : the former is characterized by abundant small islets and a somewhat large number of islets, while the latter by moderate increase in larger islets that would contribute much to the steep increase of islet volume (Table 2). The cause of the topographical difference in islet distribution remains as yet unknown. It might be attributed, as some authors suggested (Bargmann 1939; Bencosme and Liepa 1955), to some difference between the ventral and dorsal pancreatic primordia in their potentiality to form islets.

Parameters for the whole pancreas can be approximately determined by the use of histologic sections taken from the range between $x_{0.5}$ and $x_{0.7}$, or the midposition of the pancreas and the one-fourth position from the splenic end (Table 2).

\section{References}

1) Acosta, J.M., Buceta, J.C., Pons, J.E., Lodi Elena Prin de Figari, Rubio Galli, O.A.M., Weinschelbaum, E.E. \& Soloaga, J.C. (1969) Distribution and volume of the islets of Langerhans in the canine panereas. Acta physiol. latinoam., 19, 175-180.

2) Bargmann, W. (1939) Die Langerhansschen Inseln des Pankreas. In: von Möllendorff's Handbuch der mikroskopischen Anatomie des Menschen, Bd. 6, Teil 2, Springer, Berlin, pp. $197-288$.

3) Bencosme, S.A. \& Liepa, E. (1955) Regional differences of the pancreatic islet. Endocrinology, 57, 588-593.

4) Hellman, B. (1959a) The frequency distribution of the number and volume of the iselts of Langerhans in man. I. Studies on non-diabetic adults. Acta Soc. Med. upsalien., 64, 432-460.

5) Hellman, B. (1959b) Actual distribution of the number and volume of the islets of Langerhans in different size classes in non-diabetic humans of varying ages. Nature, 184, 1498-1499.

6) Lazarus, S.S. \& Volk, B.W. (1962) Quantitative studies of islets and A:B cell ratio. In: The Pancreas in Human and Experimental Diabetes, Grune \& Stratton, New York-London, pp. 25-31.

7) Maclean, N. \& Ogilvie, R.F. (1955) Quantitative estimation of the pancreatic islet tissue in diabetic subjects. Diabetes, 4, 367-376.

8) Neubert, K. (1927) Bau und Entwicklung des menschlichen Pankreas. Beitrag 12; Zur synthetischen Morphologie. Arch. f. Entwick. d. Org., 111, 29-118. Cited in 2).

9) Opie, E.L. (1900) On the histology of the islands of Langerhans of the pancreas. Bull. Johns Hopkins Hosp., 11, 205-209.

10) Suwa, N., Takahashi, T. \& Sasaki, Y. (1964) Histometrical studies of liver cirrhosis by mathematical treatments of linear intercepts on a small number of random histological sections. Tohoku J. exp. Med., 84, $\mathbf{l}-36$.

11) Suwa, N., Takahashi, T., Saito, K. \& Sawai, T. (1976) Morphometrical method to estimate the parameters of distribution functions assumed for spherical bodies from measurements on a random section. Tohoku J. exp. Med., 118, 101-111.

12) Thompson, W.R. (1932) The geometric properties of microscopic configurations. I. General aspects of projectometry. Biometrika, 24, 21-26.

13) Thompson, W.R. \& Hussey, R. (1932) The geometric properties of microscopic configurations. II. Incidence and volume of islands of Langerhans in the pancreas
of a monkey. Biometrika, 24, 27-38.

14) Warren, S., LeCompte, P.M. \& Legg, M.A. (1966) Quantitative pancreatic changes in diabetes mellitus. In: The Pathology of Diabetes Mellitus, 4th ed., Lea \& Febiger,
Philadelphia, pp. 90-101. 15) Wicksell, S.D. (1925) The corpuscle problem. A mathematical study of a
biometric problem. Biometrika, 17, 84-99. 16) Wicksell, S.D. (1926) The corpusele problem. Second memoir. Case of ellipsoidal
corpuscles. Biometrika, 18, 151-172. 